\title{
THE EFFECTIVE TEMPERATURE OF URANUS
}

\author{
G. G. Fazio, W. A. Traub, and E. L. Wright* \\ Center for Astrophysics, Harvard College and Smithsonian Astrophysical Observatories \\ F. J. Low \\ University of Arizona, Lunar and Planetary Laboratory \\ AND \\ L. TRAFTON \\ University of Texas \\ Received 1976 February 6
}

\begin{abstract}
Uranus was observed in the $40-250 \mu$ region with a $102 \mathrm{~cm}$ balloon-borne telescope, and the results were interpreted in terms of model atmosphere calculations to yield an effective temperature of $58 \pm 3 \mathrm{~K}$; this sets an upper limit of about 28 percent on the relative contribution of an internal heat source to the total thermal radiation. On the same flight we also measured the brightness temperatures of Venus $(215 \pm 13 \mathrm{~K})$ and Jupiter $(135 \pm 6 \mathrm{~K})$, thus reconfirming the existence of an internal heat source for Jupiter.
\end{abstract}

Subject headings: planets: atmospheres - planets: Jupiter — planets: Uranus - planets: Venus

\section{INTRODUCTION}

Using a balloon-borne far-infrared photometer, we have measured the thermal radiation from Uranus in a wavelength region which includes most of the expected flux. Such observations of the outer planets are of interest in that they provide information on the strength of possible internal heat sources, which in turn will place constraints on models of the interiors and on scenarios for the formation of the solar system. Although it is by now well known that Jupiter radiates about twice as much power as it receives from the Sun, the situation is less clear for the other giant planets: measurements of Saturn are difficult to interpret owing to the rings; Uranus and Neptune are not only faint, but in addition most of their thermal radiation is blocked by the Earth's atmosphere, which at ground level is essentially opaque from 35 to $300 \mu$. Previous observations of Uranus have been limited to the regions $\lambda<35 \mu$ and $\lambda>350 \mu$, each of which contains less than 2 percent of the total thermal power; the interpretation of these observations requires an accurate model atmosphere calculation in order that the measured brightness temperatures can be used to infer an effective (bolometric) temperature. In particular, observations at $\lambda \leq 35 \mu$ are sensitive to the presence of an optically thin but relatively hot inversion layer in the upper atmosphere of a planet.

\section{OBSERVATIONS}

We used the Center for Astrophysics-University of Arizona $102 \mathrm{~cm}$ diameter balloon-borne telescope in series with a far-infrared filter and an array of four

\footnotetext{
* Junior Fellow, Society of Fellows, Harvard University.
}

He-cooled Ge-Ga bolometer detectors (Fazio et al. 1974). The telescope employs a chopping secondary mirror with a beam throw of $5^{\prime}$ at a frequency of 20 $\mathrm{Hz}$; the beam size as defined by the main, central detector is $1^{\prime} \times 1.5$. The instrumental spectal response (Fig. 1) is limited at short wavelengths by a cold $\mathrm{CaF}_{2}-\mathrm{SiO}_{2}$ filter and at long wavelengths by diffraction (for point sources); estimated uncertainties in the shape of the spectral response are also indicated.

Two flights were made from Palestine, Texas, in 1975 May and June; in Table 1 we list the planets which were observed, the universal time, the polar diameters $\alpha_{p}$, the terrestrial air mass factor $\eta$, the altitude of the balloon $h$, the number of observations, the approximate signal-to-noise ratio for each observation, and the ratio of the measured power to that received from Mars. Since the bolometer sensitivity depends upon the ambient air pressure (via the helium bath temperature), we have multiplied the measured signal levels by a factor [1-0.033 $(h-28.0)]$ before forming the tabulated ratios.

The observations were made by raster scanning the sky in the neighborhood of the planet, and taking as the signal the maximum deflection which occurred when the planet was closest to being centered on the main detector. This mode of operation was dictated by the relatively large drift rate in the gyroscopes $(0,1$ per second of time). The indicated uncertainties $( \pm 1 \sigma)$ in individual flux levels are generated from errors in power measurement due to pointing, 5 percent; Mars calibration (see below), 7 percent; detector noise in the Uranus measurement, 10 percent; and filter shape uncertainties amounting to 1 percent for Venus, 2 percent for Jupiter, 4 percent for Saturn, and 7 percent for Uranus. Systematic errors are discussed in the final section of this paper. 

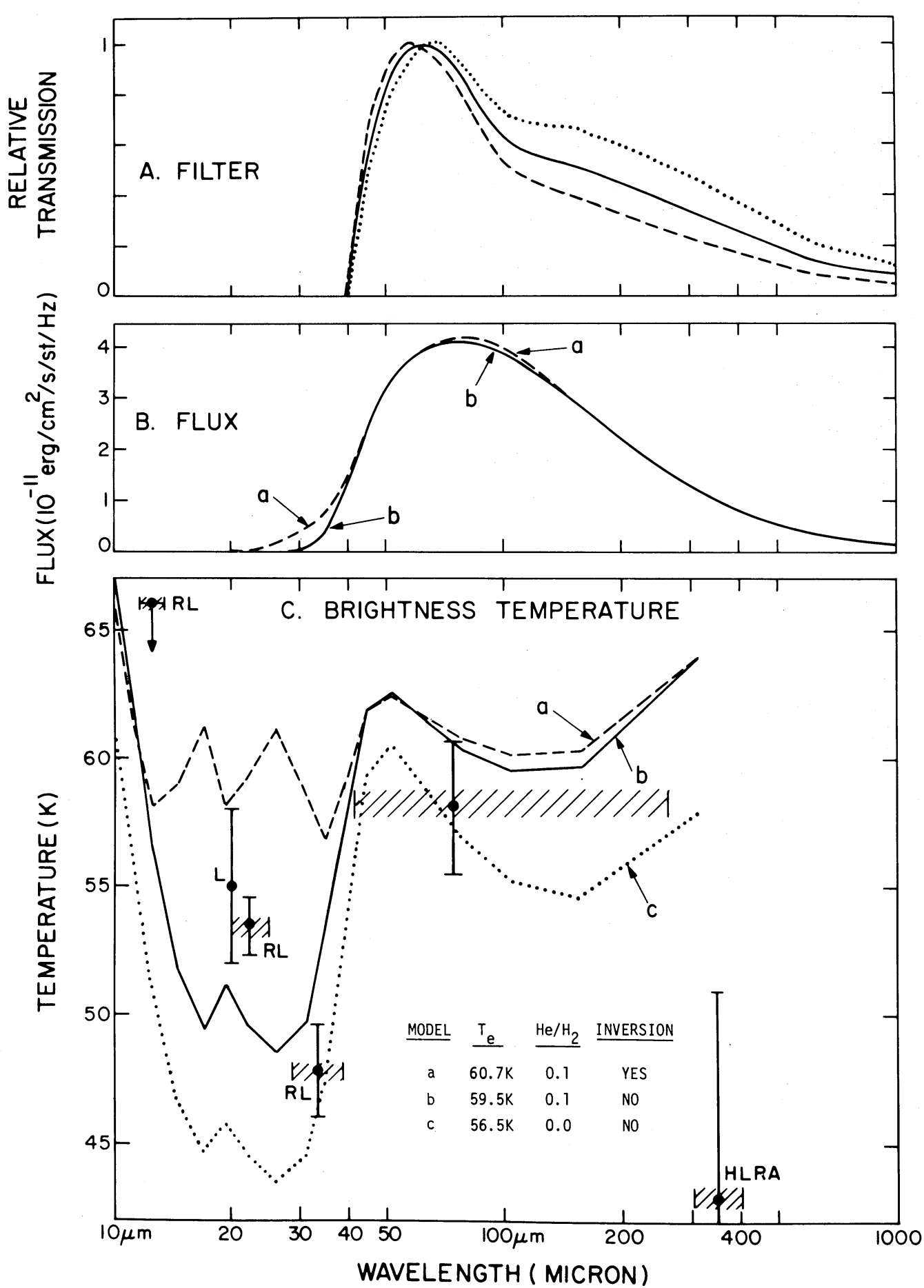

FIG. 1.- The nominal filter profile $(A)$ is shown as a solid line, with estimated uncertainties in the shape indicated by dashed lines. The effective wavelength is $71 \mu$ for Uranus. The flux $(B)$ and brightness temperature $(C)$ are shown for three of the models in our grid; see inset table for parameters. We emphasize that curves $(a, b, c)$ represent sample results from our grid of models; we estimate that the actual profile falls between curves $b$ and $c$ for $\lambda>40 \mu$, and between curves $a$ and $b$ for $\lambda<40 \mu$.

Previous observations of brightness temperature are also shown in $(C)$, along with crosshatching to indicate the spectral bandpass. The 20 to $35 \mu$ observations are primarily sensitive to a temperature inversion, as discussed in the text; these points are from Rieke and Low (1974) and Low (1966). The $350 \mu$ point from Harper et al. (1972) is only in marginal agreement with the model calculations, and suggests that there is possibly an additional source of opacity at $350 \mu$ which has not yet been accounted for. 
TABLE 1

Planetary Observations With the 40 to 250 micron Filter*

\begin{tabular}{|c|c|c|c|c|c|c|c|c|c|}
\hline $\begin{array}{c}\text { UT } \\
(1975)\end{array}$ & Planet & $\alpha_{p}(\operatorname{arcsec})$ & $\eta$ & $h(\mathrm{~km})$ & $n_{\mathrm{obs}}$ & $(\mathrm{S} / \mathrm{N})_{\mathrm{obs}}$ & $P / P_{\text {Mars }}$ & $P / P_{\text {Thermal }}$ & $T(\mathrm{~K})$ \\
\hline $\begin{array}{r}\text { May } 18 \\
3^{\mathrm{h}} 21^{\mathrm{m}} \\
3^{\mathrm{h}} 33^{\mathrm{m}}\end{array}$ & $\begin{array}{l}\text { Venus } \\
\text { Saturn }\end{array}$ & $\begin{array}{l}17.56 \\
15.42\end{array}$ & $\begin{array}{l}4.1 \\
3.9\end{array}$ & $\begin{array}{l}28.7 \\
28.7\end{array}$ & $\begin{array}{l}36 \\
24\end{array}$ & $\begin{array}{r}>10^{3} \\
10^{3}\end{array}$ & $\begin{array}{l}6.7 \pm 0.7 \\
1.4 \pm 0.2\end{array}$ & $\begin{array}{l}0.1 \\
0.5\end{array}$ & $\begin{aligned} T_{\lambda} & =192 \pm 11 \\
T_{e} & =99 \pm 6 \text { if } T_{\text {ring }}=0 \\
& =76 \pm 4 \text { if } T_{\text {ring }}=76\end{aligned}$ \\
\hline $9^{\mathrm{h}} 12^{\mathrm{m}}$ & Mars & 5.84 & 8.4 & 25.9 & 18 & $>10^{2}$ & 1.0 & 0.1 & (Calibration: $T_{\lambda} \approx 244$ ) \\
\hline $\begin{array}{l}\text { June } 16 \\
3^{\mathrm{h}} 24^{\mathrm{m}} \\
4^{\mathrm{h}} 54^{\mathrm{m}} \\
9^{\mathrm{h}} 42^{\mathrm{m}} \\
9^{\mathrm{h}} 51^{\mathrm{m}}\end{array}$ & $\begin{array}{l}\text { Venus } \\
\text { Uranus } \\
\text { Mars } \\
\text { Jupiter }\end{array}$ & $\begin{array}{r}23.16 \\
3.99 \\
6.45 \\
34.74\end{array}$ & $\begin{array}{l}3.7 \\
1.7 \\
2.7 \\
2.5\end{array}$ & $\begin{array}{l}28.7 \\
28.7 \\
27.4 \\
27.4\end{array}$ & $\begin{array}{r}48 \\
4 \\
42 \\
36\end{array}$ & $\begin{array}{l}>10^{3} \\
10 \\
>10^{2} \\
>10^{3}\end{array}$ & $\begin{array}{l}10.9 \pm 1.1 \\
0.013 \pm 0.002 \\
1.0 \\
10.9 \pm 1.1\end{array}$ & $\begin{array}{l}0.1 \\
0.7 \\
0.1 \\
0.3\end{array}$ & $\begin{array}{l}T_{\lambda}=215 \pm 13 \\
T_{e}=58 \pm 3 \\
\left(\text { Calibration: } T_{\lambda} \approx 242\right) \\
T_{e}=135 \pm 6\end{array}$ \\
\hline
\end{tabular}

* The polar diameter $\alpha_{p}$ is the same as the equatorial diameter $\alpha_{e}$ except for Saturn $\left(\alpha_{e}=1.177 \alpha_{p}\right)$ and Jupiter $\left(\alpha_{e}=1.071 \alpha_{p}\right)$. The terrestrial air mass is $\eta$, and the balloon altitude is $h$. The number of observations $\left(n_{\text {obs }}\right)$ and the approximate signal-to-noise ratio for each are indicated. The relative power observed $\left(P / P_{\text {Mars }}\right)$ is corrected to $h=28 \mathrm{~km}$, and the $1 \sigma$ standard deviation includes the estimated uncertainties of calibration, pointing, detector noise, and filter shape. Finally we list the observed fraction of the total thermal power $P / P_{\text {Thermal }}$ and the source brightness temperature $T_{\lambda}$ (for $\lambda \approx 40-250 \mu$ ) or effective temperature $T_{e}$.

\section{CALIBRATION}

Photometric calibration is derived from the measured Mars signal using an algorithm (Wright 1976) which basically consists of solving the heat-flow problem for a gray radiating surface which is periodically heated by the Sun. The model uses properties of the Martian surface which have been empirically determined at 10 and $20 \mu$ from the Mariner 6 and 7 radiometer data (Neugebauer et al. 1971); these are the thermal inertia $\left(0.006 \mathrm{cal} \mathrm{cm}^{-2} \mathrm{~s}^{-1 / 2} \mathrm{~K}^{-1}\right)$, the bolometric albedo (0.29), and the infrared emissivity $0.90(\cos \theta)^{0.1}$. The uncertainty in the calculated farinfrared brightness temperatures is about $\pm 10 \mathrm{~K}$, which amounts to a flux error of about \pm 7 percent in our bandpass. The influence of the Martian atmosphere on the soil temperature has been approximated by including a 1 percent backwarming flux. Finally, using the atmospheric transmission program of Traub and Stier (1976) as applied to the case of Mars at $200 \mathrm{~K}$ with 20 precipitable microns of $\mathrm{H}_{2} \mathrm{O}$, at an average air mass of 2 , we have calculated the atmospheric transmission in our bandpass, and find an average value of about 0.88 ; the net effect of including this absorbing and emitting layer is to reduce the calculated flux by a factor of 0.974 . There is no corresponding correction for aircraft or ground-based observations owing to the masking effect of terrestrial water vapor.

\section{URANUS}

The radiated flux of thermal power $\left(\sigma T_{e}^{4}\right)$ is inferred from the observed signal strength by the following procedure: first, we assume a theoretical emission spectrum, e.g., a Planck function, or other model, as described below; the linear diameter is taken to be $(25,900 \pm 300) \mathrm{km}$, from Danielson et al. (1973), giving an angular diameter of 3.99 arcsec; the calculated flux is multiplied by the terrestrial atmospheric transmission (mean value $\approx 0.95$ ) at each wavenumber for the actual altitude and air mass, using the results of Traub and Stier (1976); the filter response is multiplied in and the spectrum is integrated over wavenumber; finally the theoretical and measured ratios (Uranus/Mars) are compared.

As a first approximation, we take Uranus to be a blackbody, and find $T_{e}=58.3 \pm 2.2 \mathrm{~K}$. A better estimate can be made by using model calculations (Trafton 1967) for an atmosphere of $\mathrm{H}_{2}, \mathrm{He}, \mathrm{CH}_{4}$, and $\mathrm{NH}_{3}$ which is in radiative and convective equilibrium; no clouds are included, and the dominant opacity source is pressure-induced absorption by $\mathrm{H}_{2}$. We use a grid of models with various combinations of the following parameters: $T_{e} \approx 54$ to $66 \mathrm{~K}$, in steps of $3 \mathrm{~K}$; number ratio of $\mathrm{He} / \mathrm{H}_{2}=0,0.1,0.3$, and 1.0 ; methane abundances corresponding to 1 , 10 , and 100 times the solar $\mathrm{C} / \mathrm{H}$ ratio; and models with and without an upper atmospheric temperature inversion. This latter is included in order to get an idea of the effect of a possible layer that might absorb sunlight in the upper atmosphere. It would manifest itself in an enhanced brightness temperature between 10 and $40 \mu$, which is the region in which groundand aircraft-based observations are most sensitive.

For a model atmosphere with $\mathrm{He} / \mathrm{H}_{2}=0.1, \mathrm{C} / \mathrm{H}=$ solar value, and no inversion, we interpolate from our grid to find $T_{e}=56.9 \pm 2.2 \mathrm{~K}$. Variation of the $\mathrm{He} / \mathrm{H}_{2}$ ratio by \pm 0.1 only changes $T_{e}$ by roughly $\pm 0.1 \mathrm{~K}$. Regarding $\mathrm{C} / \mathrm{H}$, Wallace (1975) has suggested that $\mathrm{CH}_{4}$ is supersaturated by a factor of about 60 on Uranus; our model calculations show that even a 100-fold increase in $\mathrm{C} / \mathrm{H}$ (i.e., $\mathrm{CH}_{4}$ ) will not change the inferred $T_{e}$ by more than $0.1 \mathrm{~K}$. From Figure 1 we see that a model with $T_{e}=56.9 \mathrm{~K}$ and no thermal inversion will yield brightness temperatures which are about $9 \mathrm{~K}$ lower than is observed in the $25 \mu$ region; this amount is very nearly compensated by adding a thermal inversion, which in Figure 1 is shown only for the case of a hotter model. The effect of adding such an inversion is to increase the effective temperature by about $1 \mathrm{~K}$; thus, in round numbers, our final estimate for Uranus is $T_{e}=58 \pm 3 \mathrm{~K}$. From the 
discussion of systematic errors in the following section, we find that the temperature could be at most about $2.5 \mathrm{~K}$ warmer, using Venus as well as Mars for calibration.

The bolometric Bond albedo has been estimated to be $A^{*}=0.37 \pm 0.05$ by Dlugach and Yanovitskij (1974). Assuming that Uranus has the same temperature on its dark side as on its sunlit side (see next paragraph), we calculate (Hunten 1972) an effective temperature $T_{e}=280\left(1-\mathrm{A}^{*}\right)^{1 / 4} R^{-1 / 2}=57.0 \pm 1.1$ $\mathrm{K}$, where $R=19.18 \mathrm{AU}$. This is in good agreement with our observation, and suggests that the $(1 \sigma)$ maximum relative contribution of an internal heat source in Uranus is about 28 percent.

We assumed above that the light and dark hemispheres contribute equally to the radiated flux, although this is not obvious for Uranus, since, with its polar axis nearly in the plane of its orbit, each pole is alternately illuminated and shadowed for a period of about 42 years. If the thermal time constant were short, the temperature would vary by a factor $2^{1 / 4}$, from $57 \mathrm{~K}$ to $68 \mathrm{~K}$, between the years 1966 and 1987 ; periodic observations of $T_{e}$ would then be of considerable interest. However, it is unlikely that conditions on Uranus would permit such extreme behavior. A rough calculation of the cooling rate for a layer with $680 \mathrm{~km}$-amagat of $\mathrm{H}_{2}$ at about $77 \mathrm{~K}$ (Belton, McElroy, and Price 1971) shows that in 42 years (i.e., one-half of Uranus' year) the temperature will drop by about $6 \mathrm{~K}$ owing to radiative losses; however, upward convective transport of the hotter atmosphere below will undoubtedly provide additional heat as the upper layers cool, so the actual drop will be much less than $6 \mathrm{~K}$. A well-known example is Venus, where the dark-side temperature is very near that of the light side, and possibly even greater (Ingersoll and Orton 1974).

\section{JUPITER, VENUS, AND SATURN}

Since we are observing about 0.3 of the thermal flux from Jupiter, we may hope to equate our derived temperature with $T_{e}$. We find $T_{e}=135 \pm 6 \mathrm{~K}$, which is in reasonable agreement with three other determinations: first, the observations by Aumann, Gillespie, and Low (1969, hereafter AGL), which give $134 \pm 4 \mathrm{~K}$ for the 30 to $300 \mu$ region; second, the Pioneer 10 measurements at 20 and $45 \mu$, which give $125 \pm 3 \mathrm{~K}$ (Ingersoll et al. 1975); and third, the 30 to $300 \mu$ observations of Armstrong, Harper, and Low (1972), as recalibrated by Wright (1976, hereafter AHL/W), which yield $T_{e}=127 \pm 3 \mathrm{~K}$. Using $A^{*}=$ $0.45 \pm 0.07$ (Taylor 1965), we see that this considerably exceeds the equilibrium temperature of $106 \pm 3 \mathrm{~K}$, thus reconfirming the existence of an internal heat source (AGL).

For Venus we find $T_{\lambda}=192 \pm 11 \mathrm{~K}$ on the May flight, and $215 \pm 13 \mathrm{~K}$ on the June flight. Repeated observations of Venus during a $30 \mathrm{~min}$ period of the June flight showed no sensitivity variations greater than \pm 5 percent. These are brightness temperatures at $\lambda \approx 70 \mu$, since we are only observing about 0.1 of the total thermal flux. Comparison with the 8 to $14 \mu$ average result of $238 \mathrm{~K}$ (see discussion by Ingersoll and Orton 1974) and the 30 to $300 \mu$ temperatures $(\mathrm{AHL} / \mathrm{W})$ of $240 \pm 5 \mathrm{~K}$ shows that our values appear to be low, particularly for the May flight. Since both of these observations occurred several hours before the Mars calibrations, we have considered the possibility that the sensitivity of our measuring system increased throughout each night. Reconciliation of our Venus measurements to $240 \mathrm{~K}$ would require sensitivity increases of 46 and 20 percent on the May and June flights, respectively. We have searched for such a shift in the recorded signals from the galactic center, which was observed on both flights, and also from the blank sky offset, which is caused by temperature and emissivity differences between the sky and the telescope support structure; assuming the sky brightness to be constant, and knowing the temperature drift of the telescope, we find that there is no evidence for a gain change of more than about \pm 10 percent throughout both flights. Therefore we will continue to assume that the calibration remained constant during each flight. For reference, the expected effective temperature of Venus is $T_{e}=228 \pm 18 \mathrm{~K}$, from $A^{*}=$ $0.77 \pm 0.07$ (Irvine 1968); this is in (1 $\sigma)$ agreement with all of the $T_{\lambda}$ mentioned above, with the exception of our lowest value, from the May flight.

The Saturn measurement refers to the total emission from the disk and rings. We can obtain an upper limit on the temperature by ignoring the rings (i.e., setting $\left.T_{\text {ring }}=0\right)$; we find $T_{e}(\max )=99 \pm 6 \mathrm{~K}$. A lower limit can be obtained from the observation that the rings and disk are at approximately the same temperature (Murphy 1973; Morrison 1974) and from the calculation that on the date of observation, the projected area of the planet, plus the A and B rings, is a factor of 2.12 larger than the planet alone (based on ephemeris dimensions for the system); we can thus reduce the observed power by 2.12 and find a common temperature of $76 \pm 4 \mathrm{~K}$. This is considerably cooler than most published estimates (which range from $74 \mathrm{~K}$ to $97 \mathrm{~K}$ ), but it is interestingly close to the predicted equilibrium temperature of $78 \pm 3 \mathrm{~K}$, which is derived from a bolometric Bond albedo $A^{*}=$ $0.45(+0.12,-0.06)$ (AGL). Previous observations of Saturn and its rings (AHL/W) yield an average temperature of $85 \pm 2 \mathrm{~K}$ in the 30 to $300 \mu$ band. This corresponds to a flux which is 34 percent larger. than we observe; although this too suggests a sensitivity increase sometime during the May flight, we can find no corroborative evidence for such a shift. The present data thus constrain the ratio of Saturn's total radiated power to absorbed solar power to be in the range 1.0 to 3.3 , with the former a distinct possibility.

This work was supported by NASA grants NGR 09-015-047, NGR 22-007-270, and NGR 44-012-152.

Note added in proof 1976 June 4.-On 1976 April 8 we again observed Saturn and Mars, finding $T_{e}=$ $90 \pm 5 \mathrm{~K}$ for Saturn and its rings. This temperature is 
much more likely to be free from systematic uncertainties than the $76 \pm 4 \mathrm{~K}$ given in Table 1 above, since the observations were made by alternating between Saturn and Mars at comparable air mass factors; there was also a dc monitor on the detector, allowing us to directly determine that any possible drift in sensitivity was less than about \pm 1 percent, or $\pm 0.4 \mathrm{~K}$. This observation supports our suggestion in $\S \mathrm{V}$ above that there probably was a sensitivity change during the 1975 May flight, although the cause is still not clear.

\section{REFERENCES}

Armstrong, K., Harper, D. A., Jr., and Low, F. J. 1972, Ap. J. (Letters), 178, L89.

Aumann, H. H., Gillespie, C. M., and Low, F. J. 1969 Ap. J. (Letters), 157, L69.

Belton, M.J. S., McElroy, M. B., and Price, M. J. 1971, Ap. J., 164, 191 .

Danielson, R. E., Tomasko, M. G., and Savage, B. D. 1972, Ap. J., 178, 887.

Dlugach, J. M., and Yanovitskij, E. G. 1974, Icarus, 22, 66.

Fazio, G. G., Kleinmann, D. E., Noyes, R. W., Wright, E. L. Zeilik, M., and Low, F. J. 1974, Ap. J. (Letters), 192, L23.

Harper, D. A., Low, F. J., Rieke, G. H., and Armstrong, K. R. 1972, Ap. J'(Letters), 177, L21.

Hunten, D. M. 1972, in Physics of the Solar System, ed. S. I. Rasool (NASA SP-300).
Ingersoll, A. P., Münch, G., Neugebauer, G., Diner, D. J., Orton, G. S., Schupler, E., Schroeder, M., Chase, S. C., Ruiz, R. D., and Trafton, L. M. 1975, Science, 188, 472. Ingersoll, A. P., and Orton, G. S. 1974, Icarus, 21, 121.

Irvine, W. M. 1968, J. Atm. Sci., 25, 610.

Low, F. J. 1966, Ap. J., 146, 326.

Morrison, D. 1974, Icarus, 22, 57.

Murphy, R. E. 1973, Ap. J. (Letters), 181, L87.

Neugebauer, R., Münch, G., Kieffer, H., Chase, S. C., and Miner, E. 1971, A.J., 76, 719.

Rieke, G. H., and Low, F. J. 1974, Ap. J. (Letters), 193, L147.

Taylor, D. J. 1965, Icarus, 4, 362.

Trafton, L. M. 1967, Ap. J., 147, 765.

Traub, W. A., and Stier, M. T. 1976, Appl. Optics, 15, 364.

Wallace, L. 1975, Icarus, 25, 538.

Wright, E. L. 1976, preprint.

G. G. Fazio, W. A. Traub, and E. L. Wright: Center for Astrophysics, Harvard College Observatory and Smithsonian Astrophysical Observatory, 60 Garden Street, Cambridge, MA 02138

F. J. Low: Lunar and Planetary Laboratory, Space Science Bldg. 237, University of Arizona, Tucson, AZ 85721

L. Trafton: University of Texas at Austin, Astronomy Department, Austin, TX 78712 